\title{
AN AXIOMATIC FORMATION THAT IS NOT A VARIETY
}

\author{
KEITH A. KEARNES
}

\begin{abstract}
We show that any variety of groups that contains a finite nonsolvable group contains an axiomatic formation that is not a subvariety.
\end{abstract}

\section{INTRODUCTION}

A formation of groups is a class of finite groups closed under homomorphic images and finite subdirect products. (See [4].) The concept of a formation makes sense even if the groups are infinite, and in this paper we will use the word "formation" to refer to any class of groups that is closed under homomorphic images and finite subdirect products. Using this definition, A. Gaglione and D. Spellman ask in Problem 14.32 of The Kourovka Notebook, [6], whether every first-order axiomatizable formation of groups is a variety, i.e., an equationally axiomatizable class. They mention that the answer is affirmative for any formation of abelian groups. In this note we show that the general answer is negative.

The following result helps to put this problem into perspective.

Theorem 1.1. Let $\mathcal{V}$ be a class of similar algebraic structures. The following conditions are equivalent:

(1) $\mathcal{V}$ is a variety.

$(2) \mathcal{V}$ is closed under homomorphic images, subalgebras and products.

$(3) \mathcal{V}$ is closed under homomorphic images and subdirect products.

(4) $\mathcal{V}$ is closed under homomorphic images, subalgebras, finite products, and $\mathcal{V}$ is axiomatizable.

$(5) \mathcal{V}$ is an axiomatic formation that is closed under subalgebras.

Proof. The equivalence of (1) and (2) is Birkhoff's Theorem (see [2]). Note that these equivalent conditions easily imply each of (3)-(5).

The implication $(3) \Rightarrow(1)$ is Kogalovskiı's Theorem (see [5]).

The implication $(4) \Rightarrow(1)$ can be deduced from the theorem of van Benthem in [1] which states that an axiomatic class of algebraic structures is closed under homomorphic images and subalgebras if and only if it is axiomatizable by sentences that are finite disjunctions of equations. Thus, if (4) holds, then $\mathcal{V}$ can be axiomatized by

1991 Mathematics Subject Classification. Primary 20F17, Secondary 20E10, 08C10.

Key words and phrases. formation of groups, variety of groups. 
sentences of the form $\Phi=\Phi_{1} \vee \cdots \vee \Phi_{n}$ where each $\Phi_{i}$ is an equation. If for each $i$ there is some $\mathbf{A}_{i} \in \mathcal{V}$ that fails to satisfy $\Phi_{i}$, then $\mathbf{A}_{1} \times \cdots \times \mathbf{A}_{n}$ fails $\Phi$, which is impossible. Consequently if $\Phi=\Phi_{1} \vee \cdots \vee \Phi_{n}$ holds in $\mathcal{V}$, then for some $i$ we have that $\Phi_{i}$ also holds in $\mathcal{V}$. Hence $\Phi$ may be replaced by $\Phi_{i}$ in any axiomatization of $\mathcal{V}$. This shows that $\mathcal{V}$ is axiomatizable by equations, so (1) holds.

The implication $(5) \Rightarrow(4)$ is trivial, since a finite subdirect product is a subalgebra of a finite product.

From this result we see that the question of Gaglione and Spellman is equivalent to each of of the following questions: if an axiomatic class of groups is closed under homomorphic images and finite subdirect products, then it is closed under arbitrary subdirect products? Or: if an axiomatic class of groups is closed under homomorphic images and finite subdirect products, then it is closed under subgroups? It is the latter formulation that we will find is the easiest to use.

We close the introduction with a proof of the result of Gaglione and Spellman.

Theorem 1.2. An axiomatic formation of abelian groups is a variety.

Proof. Suppose that $\mathcal{F}$ is an axiomatic formation of abelian groups. We will prove that $\mathcal{F}$ is a variety by showing that it is closed under subgroups.

Suppose that $G \in \mathcal{F}$ and that $H$ is a subgroup of $G$. Then $G \times H \in \mathcal{F}$ for the following reason: The functions $\varphi: G \times H \rightarrow G:(g, h) \mapsto g$ and $\psi: G \times H \rightarrow$ $G:(g, h) \mapsto g-h$ are readily seen to be surjective homomorphisms. Moreover $\operatorname{ker}(\varphi)=\{(0, h) \mid h \in H\}$ and $\operatorname{ker}(\psi)=\{(h, h) \mid h \in H\}$ are disjoint. Thus $G \times H$ is a subdirect product of $(G \times H) / \operatorname{ker}(\varphi) \cong \operatorname{Im}(\varphi)=G \in \mathcal{F}$ and $(G \times H) / \operatorname{ker}(\psi) \cong$ $\operatorname{Im}(\psi)=G \in \mathcal{F}$. Since $\mathcal{F}$ contains $G$ and is closed under finite subdirect products, we get that $G \times H \in \mathcal{F}$, as claimed. Now, since $G \times H \in \mathcal{F}$ and $\mathcal{F}$ is closed under homomorphic images, we get that $H \in \mathcal{F}$ by projecting onto the second factor.

\section{Axiomatic Formations that ARE not VARIETiES}

Theorem 2.1. If $\mathcal{V}$ is a variety of groups that contains a finite nonsolvable member, then $\mathcal{V}$ contains an axiomatic subformation that is not a variety.

Proof. Choose a finite nonsolvable group $A \in \mathcal{V}$ of least cardinality. Necessarily $A$ is a finite nonabelian simple group whose proper subgroups are solvable. Let $\mathcal{V}(A)$ be the subvariety of $\mathcal{V}$ that is generated by $A$, and let $\mathcal{F}$ be the class of all groups $G$ in $\mathcal{V}(A)$ that satisfy the condition that $[N, N]=N$ for all $N \triangleleft G$. We will argue that

(i) $\mathcal{F}$ is axiomatizable,

(ii) $\mathcal{F}$ is a subformation of $\mathcal{V}$, and

(iii) $\mathcal{F}$ is not a variety.

We deal with (iii) first: $\mathcal{F}$ is not a variety since $A \in \mathcal{F}$ and no nontrivial proper subgroup of $A$ is in $\mathcal{F}$. 
For (ii), we must argue that the property that $\forall N \triangleleft G([N, N]=N)$ is inherited by homomorphic images and finite subdirect products. This property is the negation of $\exists N \triangleleft G([N, N]<N)$, hence this property is equivalent to the property that the normal subgroup lattice of $G$ has no nontrivial abelian intervals. Since the normal subgroup lattice of a homomorphic image of $G$ is isomorphic to an upper interval in the normal subgroup lattice of $G$, and abelian intervals correspond, it follows that the property we are considering is preserved by homomorphic images. Now suppose that $G \leq G_{1} \times G_{2}$ is a subdirect representation of $G$ where both $G_{1}$ and $G_{2}$ belong to $\mathcal{F}$. If $\pi_{i}: G \rightarrow G_{i}$ is the $i$-th projection, and $N_{i}=\operatorname{ker}\left(\pi_{i}\right)$, then we have that $N_{1} \cap N_{2}=\{1\}$ and that the intervals $\left[N_{i}, G\right]$ in the normal subgroup lattice of $G$ have no abelian intervals. Suppose, for the purpose of obtaining a contradiction, that $H \subsetneq K$ are normal subgroups of $G$ with $K / H$ abelian. Then $\left[N_{1} H, N_{1} K\right]$ is an abelian interval in $\left[N_{1}, G\right]$, which must be trivial, so $N_{1} H=N_{1} K$. From the modularity of normal subgroup lattice, we conclude that $\left[N_{1} \cap H, N_{1} \cap K\right]$ is a nontrivial abelian interval. Since $N_{2} \cap\left(N_{1} \cap H\right)=\{1\}=N_{2} \cap\left(N_{1} \cap K\right)$, we conclude again from modularity that $\left[N_{2}\left(N_{1} \cap H\right), N_{2}\left(N_{1} \cap K\right)\right]$ is a nontrivial abelian interval contained in $\left[N_{2}, G\right]$. There is no such interval. This contradiction proves that $\mathcal{F}$ is closed under finite subdirect products.

Finally we argue that $\mathcal{F}$ is axiomatizable. Let $\Sigma$ be a set of equations that axiomatizes $\mathcal{V}(A)$. Let $\Phi_{n}(x)$ be a first-order formula with one free variable $x$ that asserts in any group that "there exist $y_{1}, z_{1}, y_{2}, z_{2}, \ldots, y_{n}, z_{n}$ such that $x=\prod_{i=1}^{n}\left[y_{i}, z_{i}\right]$, where each $y_{i}$ and each $z_{j}$ is a product of at most $n$ conjugates of $x$ ". We show that for sufficiently large $n$ (depending on $|A|$ ) the set $\Sigma \cup\left\{\forall x \Phi_{n}(x)\right\}$ axiomatizes $\mathcal{F}$.

Assume that the group $G$ satisfies the condition $\forall N \triangleleft G([N, N]=N)$. If $g \in G$ and $N$ is the normal subgroup generated by $g$, then $g \in[N, N]$. This means that $g$ is a product of commutators $[h, k]$ where both $h$ and $k$ are products of conjugates of $g$. Thus $G \models \Phi_{n}(g)$ for some $n$. Now suppose that $G$ fails to satisfy the condition $\forall N \triangleleft G([N, N]=N)$. Then there is an $N \triangleleft G$ such that $[N, N]<N$. If $g \in N-[N, N]$, then every product of commutators $[h, k]$ with $h$ and $k$ both products of conjugates of $g$ must lie in $[N, N]$, hence $g$ is not such a product. Thus $G \not \forall \Phi_{n}(g)$ for any $n$. This shows that the condition $\forall N \triangleleft G([N, N]=N)$ is equivalent to: $\forall g \in G \exists n\left(\Phi_{n}(g)\right)$. In particular, any group satisfying $\Sigma \cup\left\{\forall x \Phi_{n}(x)\right\}$ for some $n$ belongs to $\mathcal{F}$. We now argue that there is some fixed $n$ such that every member of $\mathcal{F}$ satisfies $\Sigma \cup\left\{\forall x \Phi_{n}(x)\right\}$.

Choose $G \in \mathcal{F}$ and $g \in G$ arbitrarily. Since $G \models \Phi_{n}(g)$ for some finite $n$, there exist a finitely generated subgroup $H \subseteq G$ that contains $g$ and sufficiently many other elements so that $g$ is equal to a product of commutators of the form $\left[h, h^{\prime}\right]$ where each $h$ and $h^{\prime}$ is a product of elements that are conjugate to $g$ in $H$. Since $H$ is a finitely generated member of the locally finite variety $\mathcal{V}(A)$, it is finite. The following observation is therefore useful. 
Claim 2.2. Every finite member of $\mathcal{V}(A)$ is isomorphic to a group of the form $A^{k} \times S$ for some finite $k$ and some finite solvable group $S \in \mathcal{V}(A)$.

This can be proved by showing that the class of finite groups in $\mathcal{V}(A)$ that are isomorphic to groups of the form $A^{k} \times S$ for some finite $k$ and some finite solvable group $S \in \mathcal{V}(A)$ is closed under quotients, subgroups and products, and that this class contains the group $A$. The details are left as an exercise.

This claim shows that $H \cong A^{k} \times S$ for some finite $k$ and some solvable $S$. Without loss of generality we may assume that this isomorphism is an equality. Since $[N, N]<$ $N$ for all $N \triangleleft H$ with $N \nsubseteq A^{k} \times\{1\}$, we get that $H \models \Phi_{n}(h)$ for some $n$ and some $h \in H$ only if $h \in A^{k} \times\{1\}$. But $H \models \Phi_{n}(g)$ for some $n$ according to the choice of $H$, so $g \in A^{k} \times\{1\}$. Since $A^{k} \times\{1\} \cong A^{k} \in \mathcal{F}$, we get that $[N, N]=N$ for all $N \triangleleft A^{k}$. Thus, the subgroup $H^{\prime}=A^{k} \times\{1\}$ of $H$ contains $g$ and sufficiently many other elements so that $g$ is equal to a product of commutators of the form $\left[h, h^{\prime}\right]$ where each $h$ and $h^{\prime}$ is a product of elements that are conjugate to $g$ in $H^{\prime}$. This means that $H^{\prime}$ satisfies the defining condition for $H$, so (replacing $H$ by $H^{\prime}$ and then by an isomorphic group) there is no loss of generality in assuming that $H=A^{k}$.

Each sentence $\forall x \Phi_{n}(x)$ has the form " $\forall \exists$ (atomic)", which is Horn, hence is preserved under products. If $n$ is chosen so that $A \models \forall x \Phi_{n}(x)$, then for this same $n$ we will have $A^{k}=H \models \Phi_{n}(g)$. For this same $n$ we have $G \models \Phi_{n}(g)$ since $\Phi_{n}(x)$ is existential. Since $G \in \mathcal{F}$ and $g \in G$ were arbitrary, we get that $\mathcal{F} \models \forall x \Phi_{n}(x)$. To summarize: if $n$ is chosen so that $A \models \forall x \Phi_{n}(x)$, then every member of $\mathcal{F}$ will satisfy $\Sigma \cup\left\{\forall x \Phi_{n}(x)\right\}$. (Of course, there exists some $n$ such that $A \models \forall x \Phi_{n}(x)$ since $A \in \mathcal{F}$ and $A$ is finite. In fact, it is easy to see that $n=|A|$ is a value for which $A \models \forall x \Phi_{n}(x)$.)

\section{A POSitive EXAMPle}

The contrast between Theorem 1.2 (axiomatic formations of abelian groups are varieties) and Theorem 2.1 (a variety containing a finite nonsolvable group contains an axiomatic subformation that is not a variety) raises the question of whether every nonabelian variety of groups contains an axiomatic subformation that is not a variety. That is not the case. We show in this section now that the variety $\mathcal{N}_{2}^{p}$ of 2 -step nilpotent groups of exponent $p$, where $p$ is an odd prime, is a nonabelian variety whose axiomatic subformations are subvarieties.

Before starting, we identify the subvarieties of $\mathcal{N}_{2}^{p}$. By Corollary 35.12 of [7], any $k$-step nilpotent variety $\mathcal{V}$ is generated by its free group on $k$ generators, which we denote by $\mathbf{F}_{\mathcal{V}}(k)$. Therefore a subvariety $\mathcal{V} \subseteq \mathcal{N}_{2}^{p}$ is generated by $\mathbf{F}_{\mathcal{V}}(2)$, which is a homomorphic image of $\mathbf{F}=\mathbf{F}_{\mathcal{N}_{2}^{p}}(2)$. Collecting commutators shows that every element of $\mathbf{F}$ can be put in the form $x^{a} y^{b}[x, y]^{c}$ where $x$ and $y$ are free generators and $a, b, c \in\{0,1, \ldots, p-1\}$. Hence $\mathbf{F}$ is a nonabelian group of order $p^{3}$. Any proper homomorphic image of $\mathbf{F}$ has order dividing $p^{2}$, hence is abelian. This shows that any 
proper subvariety of $\mathcal{N}_{2}^{p}$ is abelian. Of course, any subvariety must be of exponent $p$, so the subvarieties of $\mathcal{N}_{2}^{p}$ are: the trivial variety of one-element groups, the variety of elementary abelian $p$-groups, and $\mathcal{N}_{2}^{p}$ itself. Note in particular that any nonabelian group in $\mathcal{N}_{2}^{p}$ generates the entire variety.

We now begin the task of proving that every axiomatic subformation of $\mathcal{N}_{2}^{p}$ is one of the three subvarieties that we have identified.

Lemma 3.1. Let $\mathcal{F}$ be an axiomatic subformation of $\mathcal{N}_{2}^{p}$. If $\mathcal{F}$ consists of abelian groups, then $\mathcal{F}$ is a variety.

Proof. If $\mathcal{F}$ an axiomatic subformation of the variety of abelian groups, then it is a variety by Theorem 1.2.

Lemma 3.2. Let $\mathcal{F}$ be an axiomatic subformation of $\mathcal{N}_{2}^{p}$. If $\mathcal{F}$ contains a nonabelian group, then $\mathcal{F}$ contains a finite nonabelian group.

Proof. Suppose that $G \in \mathcal{F}$ has elements $a$ and $b$ such that $[a, b] \neq 1$. Let $N$ be a normal subgroup of $G$ that is maximal with respect to not containing $[a, b]$. The group $G^{\prime}=G / N$ has noncommuting elements $a^{\prime}=a N$ and $b^{\prime}=b N$, is in $\mathcal{F}$, and is subdirectly irreducible. Changing notation back (i.e., dropping primes), we may assume that $G$ itself is subdirectly irreducible.

The center of $G$ is an elementary abelian $p$-group, which is also subdirectly irreducible since the subgroup lattice of $Z(G)$ is a lower interval in the normal subgroup lattice of $G$. This implies that $Z(G)$ is cyclic of prime order. Since $[G, G]$ is contained in the center and is not trivial, we get that $Z(G)=[G, G]$. Let $N=\langle a, b\rangle$ be the subgroup generated by our chosen noncommuting elements. Since $N$ contains the nontrivial subgroup $\langle[a, b]\rangle$, which must equal $[G, G]$, it follws that $N$ is normal. Let $C=C_{G}(N)$ be the centralizer of $N . N$ is normal and finite, so $C$ is normal and has finite index in $G$. The group

$$
K=\left\{(x, y) \in G \times G \mid x y^{-1} \in N\right\}
$$

is a subdirect product of two copies of $G$, so $K \in \mathcal{F}$. The group

$$
L=\{(x, x) \mid x \in C\}
$$

is a subgroup of $K$, which is normal since $N$ centralizes $C$. Thus $K / L \in \mathcal{F}$. We now argue that $K / L$ is finite and nonabelian.

To see that $K / L$ is nonabelian, it is enough to note that $(a, 1)$ and $(b, 1)$ are elements of $K$ whose commutator is $([a, b], 1) \notin L$. To see that $K / L$ is finite, let $\left\{g_{1}, \ldots, g_{m}\right\}$ be a transversal for $C$ in $G$. We claim that every coset of $L$ in $K$ has a representative of the form $\left(n g_{i}, g_{i}\right)$ with $n \in N$. Since $N$ is finite, and there are finitely many $g_{i}$, this will show that $K / L$ is finite. Let $(x, y) L$ be a coset of $L$. Let $n=x y^{-1} \in N$. Then $(x, y)=(n y, y)$. Now choose $g_{i}$ so that $y=g_{i} c$ for some $c \in C$. Then $(x, y)=(n y, y)=\left(n g_{i}, g_{i}\right)(c, c) \in\left(n g_{i}, g_{i}\right) L$. This establishes the claim, and completes the proof. 
Lemma 3.3. Let $\mathcal{F}$ be a formation of groups. Assume that $G \in \mathcal{F}, H$ is a subgroup of $G$, and $M$ and $N$ are normal subgroups of $G$. If

(i) $[M, N]=1$, and

(ii) $H N, H M, H M N \in \mathcal{F}$,

then $H \in \mathcal{F}$.

Proof. Let $Q$ be the subgroup of $G \times G$ that is generated by

$$
(M \times\{1\}) \cup\{(x, x) \mid x \in H N\},
$$

and let $P$ be the subgroup of $G \times G$ that is generated by

$$
(M \times\{1\}) \cup\{(x, x) \mid x \in H\} .
$$

Since $H \subseteq H N$, we get that $P \subseteq Q$. Let $\pi_{i}: G \times G \rightarrow G, i=1,2$, denote the coordinate projection homomorphisms, and also let these symbols denote the restrictions of these homomorphisms to $Q$ and $P$. Since $\pi_{1}(Q)=\langle M \cup H N\rangle=$ $H M N \in \mathcal{F}$ and $\pi_{2}(Q)=\langle\{1\} \cup H N\rangle=H N \in \mathcal{F}$, it follows that $Q$ is a subdirect product of two members of $\mathcal{F}$. Hence $Q \in \mathcal{F}$.

We now argue that $P \in \mathcal{F}$. We have $\pi_{1}(P)=\langle M \cup H\rangle=H M \in \mathcal{F}$. The group

$$
R=\{(x, x) \mid x \in N\}
$$

is easily seen to be contained in $Q$. Moreover, $R$ is a normal subgroup of $Q$, since conjugation of an element $(n, n) \in R(n \in N)$ by any generator $(m, 1)$ or $(a, a) \in Q$ $(m \in M, a \in H N)$ produces an element $\left(n^{\prime}, n^{\prime}\right) \in R$ since $[M, N]=1$ and $N \triangleleft G$. Since $Q \in \mathcal{F}$ we also have $Q / R \in \mathcal{F}$. Let $\nu$ denote the natural homomorphism of $Q$ onto $Q / R$, and also its restriction to $P$ :

$$
\nu: P \rightarrow Q / R: p \mapsto p R .
$$

We claim that $\nu$ maps $P$ onto $Q / R$. This claim is equivalent to the claim that $Q=P R$, which is obvious from the list of generators that we gave for each of $P, Q$ and $R$, We have thus produced two homomorphisms of $P$ onto members of $\mathcal{F}$, namely $\pi_{1}(P)=H M \in \mathcal{F}$ and $\nu(P)=Q / R \in \mathcal{F}$. If $(a, b) \in P$ belongs to $\operatorname{ker}\left(\pi_{1}\right)$, then $b=1$. If $(a, b) \in \operatorname{ker}(\nu)=P \cap R$, then $a=b$. Thus $\operatorname{ker}\left(\pi_{1}\right) \cap \operatorname{ker}(\nu)=\{(1,1)\}$, proving that $P$ is a subdirect product of the groups $H M, Q / R \in \mathcal{F}$. Hence $P \in \mathcal{F}$.

Since $\mathcal{F}$ is closed under homomorphic images, and $\mathcal{F}$ contains $P$, it also contains $\pi_{2}(P)=\langle\{1\} \cup H\rangle=H$. This completes the proof.

As a side observation, note that Lemma 3.3 provides another proof that an axiomatic formation consisting of abelian groups is a variety. For if $G \in \mathcal{F}$ and $H \leq G$, then take $M=N=G$ in the lemma to derive that $H \in \mathcal{F}$. Thus $\mathcal{F}$ is closed under subgroups, making it a variety.

Lemma 3.4. Let $\mathcal{F}$ be an axiomatic subformation of $\mathcal{N}_{2}^{p}$. If $\mathcal{F}$ contains a nonabelian group, then $\mathcal{F}$ contains all finitely generated groups in $\mathcal{N}_{2}^{p}$. 
Proof. From Lemma 3.2 we know that if $\mathcal{F}$ contains a nonabelian group, then it contains a finite nonabelian group $G$. We first argue that $\mathcal{F}$ also contains all subgroups of this group $G$.

If this is not the case, then let $H$ be a subgroup of $G$ that is maximal with respect to the property that $H \notin \mathcal{F}$. Choose $a \in G-H$, and let $N$ be the normal subgroup of $G$ generated by $a$. We claim that $N$ is abelian. This is equivalent to the claim that $a$ commutes with each of its conjugates $b^{-1} a b$, which is in turn equivalent to the claim that $a$ commutes with every commutator of the form $a^{-1}\left(b^{-1} a b\right)$. But commutators are central in $G$, since it is 2-step nilpotent. Hence $N$ is indeed abelian.

Apply Lemma 3.3 to this situation, taking $M=N$. We have (i) $[M, N]=1$ since $M=N$ is abelian, and also (ii) $H M=H N=H M N \in \mathcal{F}$ by the maximality of $H$ and the choice of $M$ and $N$. Lemma 3.3 yields that $H \in \mathcal{F}$, contrary to the choice of $H$. This contradiction completes the first step of the argument.

Since $G \in \mathcal{F}$ is nonabelian, the variety that it generates is $\mathcal{N}_{2}^{p}$. Therefore the finitely generated free groups of $\mathcal{N}_{2}^{p}$ are finite subdirect products of subgroups of $G$. We established in the first part of the proof of this lemma that all subgroups of $G$ belong to $\mathcal{F}$. Since $\mathcal{F}$ is closed under finite subdirect products, it therefore contains all the finitely generated free groups of $\mathcal{N}_{2}^{p}$. Since $\mathcal{F}$ is closed under homomorphic images, it follows that all finitely generated members of $\mathcal{N}_{2}^{p}$ belong to $\mathcal{F}$.

Lemma 3.5. Let $\mathcal{F}$ be an axiomatic subformation of $\mathcal{N}_{2}^{p}$. If $\mathcal{F}$ contains a nonabelian group, then $\mathcal{F}=\mathcal{N}_{2}^{p}$.

Proof. The lemma is based on the following.

Claim 3.6. Let $\mathcal{E}$ be the elementary class generated by the finitely generated free groups in $\mathcal{N}_{2}^{p}$. Each member of $\mathcal{E}$ is of the form $F \times A$ where $F$ is free in $\mathcal{N}_{2}^{p}$ and $A$ is elementary abelian. Moreover, there exist such groups in $\mathcal{E}$ where the rank of $F$ is as large as desired.

Let $F$ be a finite free group in $\mathcal{N}_{2}^{p}$. The Burnside Basis Theorem guarantees that a subset $X \subseteq F$ is a free generating set iff its image in the vector space $F /[F, F]$ is a basis. Moreover, it is easy to see from commutator collection that when $F$ is a nonabelian finite free group in this variety, then $[F, F]=Z(F)$. Thus, for all $n>1$, $F$ satisfies sentences of the form $\sigma_{n}$ : "if $\left\{x_{1}, \ldots, x_{n}\right\}$ is an independent set modulo the center, then it is a free basis of the subgroup it generates". The sentence $\sigma_{n}$ is first-order, since $n$-generated groups have bounded finite size in $\mathcal{N}_{2}^{p}$. It follows that all members of $\mathcal{E}$ satisfy all sentences $\sigma_{n}$, and therefore any member $G \in \mathcal{E}$ also has the property that a subset $Y \subseteq G$ freely generates a subgroup if it is independent modulo the center. By the Compactness Theorem, for any infinite cardinal $\kappa$ there exists a group $G \in \mathcal{E}$ with a subset $Y \subseteq G$ of size $\kappa$ that is independent modulo the center. By enlarging $Y$ if necessary, we may assume that $Y$ is independent modulo the center and $Y / Z(G)$ is a basis of $G / Z(G)$. In this case $Y$ generates a free subgroup 
$F \leq G$ that intersects each coset of $Z(G)$. Now $F \cap Z(G)$ is a subspace of the vector space $Z(G)$, hence has a complement $A$ in this space. We have $F \cap A=\{1\}$ by the choice of $A$, and $F A=G$ since $F$ contains a transversal for $Z(G)$ and $F A$ contains $Z(G)$. Thus $G \cong F \times A$ where $F$ is free of rank at least $\kappa$ and $A$ is elementary abelian. Since $\kappa$ was an arbitrary infinite cardinal, this establishes both parts of the claim.

To complete the proof of this lemma assume that $\mathcal{F}$ is an axiomatic subformation of $\mathcal{N}_{2}^{p}$ that contains a nonabelian group. By Lemma 3.4, $\mathcal{F}$ constains all the finitely generated groups in $\mathcal{N}_{2}^{p}$. Since $\mathcal{F}$ is axiomatic, it therefore contains $\mathcal{E}$. Claim 3.6 shows that $\mathcal{F}$ contains groups of the form $F \times A$ where $F$ is free in $\mathcal{N}_{2}^{p}$ of arbitrarily large rank. Any group in $\mathcal{N}_{2}^{p}$ is a quotient of a group of the form $F \times A$ provided the rank of $F$ is large enough, since any group in $\mathcal{N}_{2}^{p}$ is a quotient of some free $F$ provided the rank is large enough. Thus $\mathcal{F}$ contains all of $\mathcal{N}_{2}^{p}$.

Lemmas 3.1 and 3.5 combine to yield the result that every axiomatic subformation of $\mathcal{N}_{2}^{p}$ is a variety.

One might imagine that an axiomatic formation generated by the finite members of a locally finite variety would be forced to contain the infinite members of the variety. If this were true, then our argument could have ended with Lemma 3.4. But notice that the axiomatic formation generated by the finite members of the variety of meet semilattices consists only of semilattices with a least element, hence does not contain all the infinite members of the variety. Thus Lemma 3.5 is really an essential part of the argument.

We close this paper with a refinement of the question of Gaglione and Spellman.

Question 3.7. Which (finitely generated) varieties of groups have the property that all axiomatic subformations are subvarieties?

\section{REFERENCES}

[1] J. van Benthem, A note on Jónsson's theorem, Algebra Universalis 25 (1988), 391-393.

[2] G. Birkhoff, On the structure of abstract algebras, Proc. Cambridge Philos. Soc. 31 (1935), 433-454.

[3] S. Burris and H. P. Sankapannavar, A Course in Universal Algebra, Graduate Texts in Mathematics 78, Springer-Verlag, New York, 1981.

[4] W. Gaschütz, Zur Theorie der endlichen auflösbaren Gruppen, Math. Z. 80 (1962/1963), 300305.

[5] S. R. Kogalovskiŭ, On Birkhoff's theorem, (Russian) Uspehi Mat. Nauk 20 (1965) no. 5 (125), 206-207.

[6] The Kourovka notebook. Unsolved problems in group theory, Fourteenth augmented edition. Edited by V. D. Mazurov and E. I. Khukhro. Russian Academy of Sciences Siberian Division, Institute of Mathematics, Novosibirsk, 1999.

[7] H. Neumann, Varieties of Groups, Springer-Verlag New York, Inc., New York, 1967. 
(Keith Kearnes) Department of Mathematics, University of Colorado, Boulder, CO 80309-0395, USA

E-mail address: kearnes@euclid.colorado.edu 